\title{
The Concept and Necessity of Learning Outcome
}

\section{Sun Kim}

Department of Medical Education, The Catholic University of Korea School of Medicine, Seoul, Korea

\section{학습성과의 개념과 필요성}

가톨릭대학교 의과대학 의학교육학과

\section{김 선}

의학교육은 사회에서 요구하는 바람직한 의사 양성과 직결 되어 있기 때문에 사회의 시대적 변화 흐름을 간과할 수 없으 며, 사회가 요구하는 의사상은 무엇인지를 파악하여 교육에 반영해야 할 것이다. 현대사회가 정보화 시대로 접어들고 빠 른 속도로 변하면서 교육에서는 수많은 지식을 주입하는 것 은 더 이상 의미가 없게 되었다. 필요한 지식에 접근하는 방법 이 매우 다양해짐에 따라 언제 어디서든 찾아 볼 수 있게 되었 기 때문이다. 교육에서 더 중요한 것은 기본 지식을 실제 상황 에 적용하고 활용하여 문제해결능력, 의사결정능력, 판단력 등과 같은 수행능력을 함양하는 것에 있다고 볼 수 있다. 같은 맥락에서 의학교육에서도 많은 지식을 갖춘 의사보다는 수행 할 수 있는 능력과 자질을 갖춘 의사를 요구한다. 그러나 그 동안의 의학교육에서는 역량 함양보다는 지식을 가르치는 데 주력해 왔다는 지적이 있다[1,2]. 따라서 진료현장에서 지식 을 활용하여 실제 수행을 할 수 있는 의사 양성을 위해서는 역량 교육에 초점을 두는 학습성과의 개념이 중요하게 되었 다. 학습성과의 개념을 의학교육에 반영할 수 있도록 국내외 의학교육 기관에서는 학습성과와 관련된 보고서를 발간하였
다. 미국의 경우 1984 년에 General Professional Education of Physician (GPEP) [3]와 1998년에 American Association of Medical Colleges (AAMC)에서 발간된 Medical School Objective Project (MSOP) [4]를 들 수 있다. GPEP 보고서에서는 그 동안의 의학교육은 지식 위주의 주입식 교 육이었음을 지적하면서 전문직 의사 양성 교육에서는 의학지 식에 준하는 술기와 태도를 갖춘 역량 있는 의사를 양성해야 한다고 비판하고 있다. $\mathrm{MSOP}$ 보고서에서는 의과대학생이 갖추어야 할 구체적인 학습목표를 성과의 개념으로 제시함으 로써 의과대학 교육과정 개발과 개선에 반영할 수 있도록 지 침을 제공하고 있다. 영국에서는 General Medical Council (GMC)에서 1993년[5], 2003년[6], 2009년[7]에 걸쳐 '미래의 의사상이라는 보고서를 발간하고 있는데[3], 이 보고서에는 의과대학생들에게 요구되는 매우 구체적인 성과를 제시하여 모든 대학이 이를 교육과정에 반영하도록 하고 있다. 우리나 라에서도 한국의과대학장협의회(현 한국의과대학장협회)에 서 21세기 한국의학교육계획이라는 보고서를 발간하여[8] 바 람직한 의사양성 교육을 위한 구체적인 방향과 성과를 제시
Received: May 7, 2012 • Revised: May 14, 2012 • Accepted: May 17, 2012 Corresponding Author: Sun Kim

Department of Medical Education, The Catholic University of Korea School of Medicine, 222 Banpo-daero, Seocho-gu, Seoul 137-701, Korea

Tel: +82.2.2258.7200 Fax: +82.2.591.7185 email: skm@catholic.ac.kr
Korean J Med Educ 2012 Jun; 24(2): 89-92. http://dx.doi.org/10.3946/kjme.2012.24.2.89 pISSN: 2005-727X eISSN: 2005-7288

(C) The Korean Society of Medical Education. All rights reserved. This is an open-access article distributed under the terms of the Creative Commons Attribution Non-Commercial License (http:// creativecommons.org/licenses/by-nc/3.0/), which permits unrestricted non-commercial use, distribution, and reproduction in any medium, provided the original work is properly cited. 
하고 있다. 최근 이와 같은 학습성과 중심 교육을 장려하고 대 학의 교육과정에 제대로 반영되고 있는지를 평가하기 위해 한국의학교육평가원이 인증기준에 학습성과 관련 기준을 도 입하고 있는 것도 학습성과의 중요성이 반영된 결과라고 할 수 있다. 의학교육에서 학습성과는 세계적으로 관심을 모으 고 있으며, 우리의 의학교육에서도 더 이상 간과할 수 없게 되 었다. 따라서 이 글에서는 의학교육자에게 학습성과에 대한 기본적인 이해를 위해 성과의 개념은 무엇인지, 도입 배경이 된 이론의 소개, 학습목표와의 차이점 등을 간략하게 살펴보 고자 한다.

\section{학습성과의 개념과 도입 배경}

학습성과의 개념에서는 교육의 결과가 과정을 결정한다는 관점을 가지고 있으며, 이는 학생이 교육을 마친 후 구체적으 로 보여주어야 하는 역량이 무엇인지를 밝히는 것의 의미라 고 할 수 있다. 따라서 학습성과 중심 교육에서는 학생이 무엇 을 학습하기를 원하는지, 왜 그것을 배워야 하는지, 교수는 학 생이 그것을 배우기 위해 어떤 도움을 줄 수 있는지, 그리고 학생이 배웠다는 것을 어떻게 확인할 수 있는지 등을 고려해 야 한다. 이런 의미에서 학습성과는 교수자가 학생들에게 기 대하는 것이 무엇인지 분명하게 말할 수 있도록 하는 것이며, 학습성과 도달을 위한 교육 내용, 방법, 평가를 선택하는 기준 이 된다. 또한 이와 같이 명확한 학습성과는 학생에게는 효과 적으로 학습하고, 현재 자신의 성과와 수준을 확인하는 데 도 움을 줄 수 있는 것이다. 학습성과의 위계도 또는 수준을 살펴 보면 크게 4단계로 구분할 수 있으며[9], 1단계 수준은 졸업 성과로서 의과대학생이 기본의학교육을 마치고 졸업 시 갖추 고 있어야 할 항목들로, 대학의 교육목표를 세분화하여 성과 의 개념으로 표현한 것을 의미한다. 2 단계 수준은 시기별 학 습성과로서 졸업성과를 각 시기별로 세부화한 것으로, 이는 대학에서 자체적으로 구분한 시기별(예: 학년별 또는 기초의 학, 임상의학, 임상실습 시기 등) 학습성과를 의미한다. 3 단계 수준에서의 성과는 단위과정별 학습성과이며, 이는 시기별 학습성과를 보다 세분화한 것으로 예를 들면, 인체의 정상구 조, 성장과 노화, 소화기, 호흡기 등과 같은 단위과정별로 구
분된 학습성과를 의미한다. 마지막 4단계 수준의 학습성과는 수업성과로서 단위과정별 학습성과를 각 수업별로 세분화한 것으로 개별 수업에 적용되는 학습성과를 의미한다.

이와 같은 학습성과의 개념은 아주 새로운 것은 아니며, 1949년 잘 알려진 교육학자 R. Tyler의 이론으로 거슬러 올 라갈 수 있다[10]. Tyler는 교육은 인간의 행동양식을 변화시 키는 과정으로 보았으며, 이런 의미에서 학습목표는 학습과 정을 통해 학생들에게 나타나는 행동의 변화라고 보았다. 당 시 Tyler는 학습성과의 개념이 아닌 학습목표라는 개념을 사 용하면서 학습목표의 이원분류표를 제시하였다. Tyler의 이 원분류 학습목표는 학생이 학습해야 할 내용과 학습의 결과 로 나타나야 할 행동이라는 이차원적 구조를 갖고 있는 것이 특징이다. 여기에서 행동으로 표현된 목표가 바로 최근 사용 되고 있는 학습성과의 개념과 유사한 개념으로 볼 수 있다.

1960년대에는 Glaser의 준거지향평가의 개념이 도입되었 는데, 이는 학생의 학업성취 수준을 양적으로 측정하는 것에 서 질적 평가를 강조하는 것이다[11]. 양적인 평가를 강조하 는 기준지향평가와는 달리 질적 평가는 학생들의 성취도를 "누가 누가 잘하나"라고 하는 상대적 서열을 비교하는 의미 보다는 개개인의 학생이 무엇을 얼마나 잘 알고 있는지를 강 조하는 것이 특징이다. 이런 의미에서 학생이 학습성과를 얼 마만큼 도달했는지의 여부를 확인하는 것은 준거지향 평가의 개념에 기반하고 있다고 볼 수 있다.

한편 학습방법의 관점에서 보면 1960년대에 Bloom의 완전 학습(mastery learning) 개념이 소개되었는데, 이는 학습목 표를 전체 학생의 $90 \%$ 이상이 완전히 수행하는 학습을 의미 한다[12]. 결국 완전학습은 모든 학생이 정해진 학습목표를 도달하는 것을 의미하는 차원에서 질적 평가와 성과의 개념 과 유사하다고 할 수 있다.

\section{학습목표와 학습성과의 차이점}

많은 의학교육자들이 학습성과의 개념이 기존에 사용되었 던 학습목표와는 어떤 구체적인 차이가 있는지에 대한 의문 을 가지고 있는 것이 사실이다. 두 가지의 개념은 어떠한 관점 으로 교육을 보는지에 대한 인식의 차이가 더 크다고 할 수 
있다. 학습목표와 학습성과의 개념 정립을 위해 몇 가지 구체 적인 차이점을 살펴보면 다음과 같다. 학습목표는 가르치는 사람의 입장이 강조가 되는 반면 학습성과에서는 배우는 사 람, 즉 학습자의 관점이 강조가 된다. 교수의 입장에서 학습목 표 도달을 위해 어떤 내용을 가르칠 것인가 와는 달리 학습자 중심교육에서는 학습자가 무엇을 할 수 있어야 하는지, 어떤 과업을 해 낼 수 있어야 하는지에 초점을 두는 것이다. 학습목 표가 가르쳐야 할 내용 중심이라면, 학습성과는 학생이 갖추 어야 할 지식의 내용보다는 이를 바탕으로 실제로 수행할 수 있는 어떤 역량을 갖추어야 하는지를 더 중요하게 다루는 과 업중심이라고 할 수 있다. 내용중심 또는 교수자중심에 초점 을 두는 학습목표는 지식의 투입을 강조하는 반면 학습성과 에서는 지식을 기반으로 어떤 결과가 도출되는가에 초점을 주는 결과중심 접근방법이다[13].

학생의 성취도를 판단하는 과정에서도 학습목표의 개념에 서는 목표의 도달 여부를 학생 간 상대적인 평가를 통하여 확 인하는 것을 중요하게 생각하는 반면, 학습성과 개념에서는 학생의 상대적 서열보다는 모든 학생이 학습성과를 어떤 수 준으로 도달하는지에 더 관심을 두는 질적인 평가를 선호한 다. 따라서 학습성과의 개념에서는 최종적인 종합평가보다는 학생의 수행 수준을 확인하고 피드백을 제공해 주는 형성평 가를 더 중요하게 다루고 있다.

학습목표 또는 성과의 개념의 사용에 있어 Prideaux는 이 러한 개념을 인위적으로 구분하는 일은 매우 어려운 과정이 기 때문에 오히려 개념의 정립을 위해 다음과 같은 질문이 중 요하다고 강조한다[14].

1) 의학교육에서는 어떤 성과가 의미 있고 지속되어야 하는가?

2) 이와 같은 성과가 교육과정에 반영되어 있는지 어떻게 확인해야 하는가?

3) 복잡하고 표현하기 어려운 학습성과도 포함되어 있는지 어떻게 확인해야 하는가?

4) 선정된 성과를 도달하기 위한 학습경험은 적절한지 확인 할 수 있는가?

\section{결론}

의학교육은 사회에서 요구하는 바람직한 의사 양성 교육을 위해 지속적으로 사회변화를 확인하여 시대의 흐름에 적절한 대처를 해야 할 것이다. 최근 우리 사회는 많은 것을 아는 의 사보다는 실제로 수행할 수 있는 의사를 원하고 있기 때문에 의학교육도 수행능력과 역량 함양에 초점을 두는 교육의 실 천을 위한 노력을 취해야 한다. 실제로 수행할 수 있는 의사 양성을 위해서는 기존의 지식 위주의 학습목표에서 과업중심 의 학습성과를 개발할 필요가 제기되고 있으며, 학습성과의 개념은 최근 의학교육의 추세인 학생중심 교육을 지향하고 있는 점에서도 교육과정에 반영되어야 할 필요가 충분히 있 다고 본다.

의과대학은 주기적으로 교육과정을 검토하고 개선해야 하 는데, 이때 학습성과의 개념을 바탕으로 보다 실제적이고 역 량을 함양하는 의학교육이 될 수 있도록 기여하기를 바란다.

Acknowledgements: None.

Funding: None.

Conflicts of interest: None.

\section{REFERENCES}

1. Kim YL. 21segie gidaehaneun uisasang. J Korean Med Assoc 2000; 43: 37-44.

2. Meng KH. Teaching medical professionalism in Korean medical schools: tasks and prospect. Korean J Med Educ 2008; 20: 3-10.

3. Association of American Medical Colleges. Physicians for the twenty-first century. The GPEP report: report of the panel on the general professional education of the physician and college preparation for medicine. Washington DC, USA: Association of American Medical Colleges; 1984. 4. Association of American Medical Colleges. Report I. 
Learning objectives for medical student education: guidelines for medical schools. Washington DC, USA: Association of American Medical Colleges; 1998.

5. General Medical Council. Tomorrow's doctors: recommendations on undergraduate medical education. London, UK: General Medical Council; 1993.

6. General Medical Council. Tomorrow's doctors: recommendations on undergraduate medical education. London, UK: General Medical Council; 2003.

7. General Medical Council. Tomorrow's doctors: outcomes and standards for undergraduate medical education. London, UK: General Medical Council; 2009.

8. The Korean Council of Deans of Medical College. Medical education plan for 21 st century. Seoul, Korea: The Korean Council of Deans of Medical College; 1999. 9. Harden RM, Crosby JR, Davis MH, Friedman M. AMEE guide no. 14: outcome-based education. Part 5. From competency to meta-competency: a model for the specification of learning outcomes. Med Teach 1999; 21: 546-552.

10. Tyler RW. Basic principles of curriculum and instruction. Chicago, USA: University of Chicago; 1949.

11. Glaser R. Instructional technology and the measurement of learing outcomes: some questions. Am Psychol 1963; 18: 519-521.

12. Bloom BS. Learning for mastery. Eval Comment 1968; 1: $1-12$.

13. Harden RM. Learning outcomes and instructional objectives: is there a difference? Med Teach 2002; 24: 151155.

14. Prideaux D. The emperor's new clothes: from objectives to outcomes. Med Educ 2000; 34: 168-169. 